\title{
Cerebral Blood Flow Volume Using Color Duplex Sonography in Patients With Fibromyalgia Syndrome
}

\author{
Arzu KAYA, ${ }^{1}$ Gürkan AKGÖL, ${ }^{1}$ Arif GÜLKESEN, ${ }^{1}$ Ahmet Kürşad POYRAZ, ${ }^{2}$ \\ Tülay YILDIRIM, ${ }^{3}$ Murad ATMACA ${ }^{4}$ \\ ${ }^{1}$ Department of Physical Medicine and Rehabilitation, Medical Faculty of Firat University, Elazig, Turkey \\ ${ }^{2}$ Department of Radiology, Medical Faculty of Firat University, Elazig, Turkey \\ ${ }^{3}$ Department of Physical Medicine and Rehabilitation, Medical Faculty of Inonu University, Malatya, Turkey \\ ${ }^{4}$ Department of Psychiatry, Medical Faculty of Firat University, Elazig, Turkey
}

\begin{abstract}
Objectives: This study aims to evaluate cerebral blood flow using color duplex Doppler ultrasonography in patients with fibromyalgia syndrome (FMS).

Patients and methods: The study included 30 female patients with FMS (mean age 42.3 years; range 22 to 59 years) and 30 female healthy controls (mean age 39.6 years; range 22 to 56 years). Color duplex Doppler ultrasonography imaging was performed with an EPIQ 5 unit equipped with a multi-frequency linear probe (3-12 MHz) in the supine position. Severity of pain, fatigue, and the patient's and physician's global assessments of disease were evaluated on a visual analog scale. The Symptom Severity Scale, Hamilton Anxiety Rating Scale, Hamilton Depression Evaluation Scale, and Fibromyalgia Impact Questionnaire were also implemented to assess disease severity.

Results: Cerebral blood flow volume and bilateral internal carotid artery (ICA) and vertebral artery (VA) volumes were not significantly higher in FMS patients compared to controls. Bilateral ICA and VA diameters were similar between FMS patients and controls. Bilateral mean peak systolic velocities and end diastolic velocities in the common carotid arteries, ICAs and VAs were similar in both groups. A significant correlation between symptom severity parameter and the cerebral blood flow volume was noted in FMS patients.

Conclusion: Cerebral blood flow volume, ICA flow, and VA flow do not appear to increase, and are correlated with only Symptom Severity Scale among other clinical parameters reflecting disease severity in patients with FMS.

Keywords: Cranial flow; depression; color duplex Doppler ultrasonography; fibromyalgia; pain.
\end{abstract}

Fibromyalgia syndrome (FMS) is a disorder characterized by widespread musculoskeletal pain, accompanied by fatigue, sleep disturbance, anxiety, and mood disorders. ${ }^{1}$ Paresthesia, cognitive difficulties, dizziness, weakness, and a subjective feeling of swelling are also frequent symptoms of the disease. Notably, in individuals with FMS, there is a widespread and immediate alteration in the sympathetic/ parasympathetic balance. Furthermore, anxiety, which generally leads to misdiagnosis, is very common in fibromyalgia, and has been found to be related to the severity of pain in FMS. ${ }^{2}$ Despite considerable attention and clinical investigation, the mechanisms responsible for initiating and perpetuating FMS remain unknown or poorly understood. In the past decade, multiple advances in neuroimaging have been applied as a means of investigating potential causative mechanisms in FMS.

In neuropsychiatric research, cerebral blood flow (CBF) has been used as an indicator of brain function. ${ }^{3}$ Historically, research on $\mathrm{CBF}$ was seriously limited by the invasive nature of the available measurement techniques, such as

Received: April 13, 2017 Accepted: April 26, 2017 Published online: October 13, 2017

Correspondence: Arzu Kaya, MD. Fırat Üniversitesi Tıp Fakültesi Fiziksel Tıp ve Rehabilitasyon Anabilim Dalı, 23119 Elazığ, Turkey.

Tel: +90 424 - 2333555 / 2031 e-mail: drarzukaya26@hotmail.com

(2018 Turkish League Against Rheumatism. All rights reserved. 
nitrous oxide inhalation and intracarotid injection techniques. ${ }^{4}$ However, recently, regional changes in brain perfusion and metabolism have been documented through the use of positron emission tomography and single photon emission computed tomography..$^{5-7}$ In addition, transcranial and extracranial color duplex Doppler ultrasonography are non-invasive techniques that are simple to administer, and may constitute an acceptable alternative for the evaluation of cerebral hemodynamic changes. Modern duplex scanners allow determination of the blood flow volume in an extracranial vessel by combination of mean blood flow velocity and vessel diameter or cross sectional area assessments. ${ }^{8}$ A parallel relationship between CBF and non-specific arousal, as mediated by the brainstem reticular activating system, has previously been reported. ${ }^{9}$ It is thought that non-specific arousal is a biological substrate for anxiety..$^{10}$ In contrast, anxiety-related factors, including sympathetic stimulation, increased blood viscosity, and reduced carbon dioxide levels, have been shown to decrease CBF. ${ }^{11}$ Thus, anxiety is associated with both cerebral vasodilatory and vasoconstrictive mechanisms. It is not surprising that investigators have observed contrasting results for anxiety-related changes in $\mathrm{CBF} .{ }^{12}$

When taking into consideration that FMS is closely related to psychogenic factors and alterations of functions of the autonomous nervous system, we hypothesized that a number of alterations may take place in blood flow rates through the internal carotid artery (ICA) and vertebral artery (VA) in patients with FMS, in keeping with intracranial vascular hemodynamic changes reported previously. ${ }^{10,13-15}$ We hypothesized that: (i) stronger changes of ICA and VA blood flow velocities would be observed in patients with FMS than in healthy controls, and (ii) that a positive association between the magnitude of the hemodynamic response and the severity of clinical pain, depression, and anxiety would be observed. Therefore, in this study, we aimed to evaluate CBF using color duplex Doppler ultrasonography in patients with FMS.

\section{PATIENTS AND METHODS}

The study, which was conducted at Firat University Hospital between January 2015 and November
2015, included 30 female patients with FMS (mean age 42.3 years; range 22 to 59 years) and 30 female healthy controls (mean age 39.6 years; range 22 to 56 years). All FMS patients met the 1990 and 2010 American College of Rheumatology classification criteria for FMS.16,17 Blood pressure measurement was performed prior to sonographic evaluation. Patients with a systolic blood pressure $<140 \mathrm{mmHg}$ and a diastolic blood pressure $<90 \mathrm{mmHg}$ were eligible for inclusion. Patients with systemic inflammatory rheumatic diseases; acute diseases and/or conditions leading to clinical confusion (fracture, malignity etc.); cooperation problems; hypertension; diabetes mellitus and other endocrinologic diseases; alcohol and drug addictions; ICA plaques; cerebrovascular diseases; chronic neurological diseases cardiac failure; a psychiatric disorder involving a history of psychosis, current suicide risk or attempt within the past two years, left-handedness or who were pregnant were excluded. The study protocol was approved by the Firat University Faculty of Medicine Ethics Committee. All the participants consented to participant. The study was conducted in accordance with the principles of the Declaration of Helsinki.

Severity of pain, fatigue, and the patient's and physician's global assessments of disease were assessed on a visual analog scale. Algometric assessment was performed for patients with fibromyalgia, with the tender point count and chronic widespread pain duration recorded. The Symptom Severity (SS) scale ${ }^{17}$ and Fibromyalgia Impact Questionnaire (FIQ) scores were obtained in all subjects. Hamilton Anxiety Rating Scale (HAM-A) and Hamilton Depression Evaluation Scale (HAM-D) were also administered. For the HAM-A scale, scores between 6 and 14 points and scores greater than 15 were interpreted as minor and major anxiety, respectively. When analyzing responses from the HAM-D scale, scores between 8 and 17 points, between 18 and 24 , and above 25 were defined as mild, moderate, and severe depression, respectively. ${ }^{18,19}$

Routine outpatient tests, including complete blood count, erythrocyte sedimentation rate, serum $\mathrm{C}$-reactive protein, rheumatoid factor, alanine aminotransferase, aspartate aminotransferase, creatine kinase, calcium, phosphorus, parathormone, vitamin $\mathrm{D}$, and thyroid stimulating hormone levels, were assessed. 
Color duplex Doppler ultrasonography imaging was performed with an EPIQ 5 unit (Philips Healthcare, Bothell, WA, USA) equipped with a multi-frequency linear probe $(3-12 \mathrm{MHz}$ ) for both patient groups, in the supine position. The entirety of the common carotid arteries, carotid bifurcations, ICAs (as distal as possible), and VAs were scanned in transverse and longitudinal planes with both the B-mode and color Doppler mode. Flow velocities and flow volumes were measured from the common carotid arteries in the center stream, approximately $2 \mathrm{~cm}$ below the bifurcation, on the grayscale $\mathrm{B}$-mode image. Internal carotid artery measurements were made approximately $2 \mathrm{~cm}$ above the bifurcation, beyond the bulb widening. The VA measurements were taken from intravertebral segments of the artery. Measurements were made with a constant $60^{\circ}$ insonation angle, minimum wall filter, and a transmitter output of $100 \%$. Peak systolic velocity, end diastolic velocity, flow volume, and luminal diameters were noted. All study examinations were performed by the same radiologist with 10 years of experience. Patients with plaques and hypoplastic VAs were excluded.

After detection of the ICA and VA flow volumes, $\mathrm{CBF}$ volume was calculated as the sum of flow volumes in the ICA and VA of both sides. The complete examination took approximately 15 minutes for each subject.

\section{Statistical evaluation}

Statistical analysis was performed using the IBM SPSS Statistics for Windows, version 20.0 (IBM Corp., Armonk, NY, USA). KolmogorovSmirnov test was used to evaluate whether variables had normal distribution. Results were evaluated using parametric and nonparametric statistical methods for data with normal and non-normal distribution, respectively. Intergroup comparisons were performed using independent group $t$ tests for parametric values, and Mann-Whitney $U$ tests for nonparametric values. Categorical data were analyzed by the Chi-square test. Correlations between parameters were evaluated using Pearson correlation analysis. In statistical evaluation, $\mathrm{p}<0.05$ was taken to indicate significance. Results were given as mean \pm standard deviation.

\section{RESULTS}

There was no statistically significant difference between FMS patients and controls in terms of age. Body mass index and marital status were similar in both groups. The mean disease duration was $2.9 \pm 1.8$ years in the FMS group. Coexistence of anxiety was observed in 21 fibromyalgia patients (70\%) and one control (according to HAM-A evaluation), while depression was noted in 15 fibromyalgia patients (50\%) and one control (3.3\%) (according to HAM-D evaluation) (Table 1).

Fibromyalgia Impact Questionnaire, SS scale, HAM-A, and HAM-D parameters were significantly higher in FMS patients than controls. Similarly, the patient's and physician's global pain evaluation parameters were significantly higher in FMS patients $(p<0.001)$ (Table 1).

Cerebral blood flow volume and bilateral ICA and VA volumes were not significantly higher in FMS patients compared to controls. Bilateral ICA and VA diameters were similar between FMS patients and controls. Furthermore, mean peak systolic and end diastolic velocity of bilateral common carotid arteries, ICAs and VAs did not differ between FMS patients and controls (Table 2).

There was significant correlation between FIQ and the results of either HAM-A or HAM-D in FMS patients $(r=0.693, p=0.001$ vs. $r=0.378$, $p=0.04$, respectively). A significant correlation between SS parameter and the CBF volume in FMS patients was noted $(r=0.376, p=0.041)$. However, there was no statistically significant correlation between other clinical parameters (FIQ, chronic widespread pain duration, HAM-A, HAM-D) and radiological data.

\section{DISCUSSION}

This study aimed to investigate whether CBF was related to the processing of pain in patients with fibromyalgia. To our knowledge, this is the first study in which CBF volume has been assessed using extracranial duplex sonography in patients with FMS. We found no significant differences in CBF volume and ICA flow velocity in patients with FMS, when compared with healthy controls. 
Table 1. Comparisons of demographic and clinical parameters between fibromyalgia syndrom patients and healthy control groups

\begin{tabular}{|c|c|c|c|c|c|c|c|}
\hline & \multicolumn{3}{|c|}{ Fibromyalgia syndrome patients $(n=30)$} & \multicolumn{3}{|c|}{ Healthy controls $(n=30)$} & \multirow[b]{2}{*}{$p$} \\
\hline & $\mathrm{n}$ & $\%$ & Mean \pm SD & $\mathrm{n}$ & $\%$ & Mean \pm SD & \\
\hline Age (years) & & & $42.3 \pm 8.5$ & & & $39.6 \pm 9.6$ & 0.252 \\
\hline Duration of the disease (years) & & & $2.9 \pm 1.8$ & & & & \\
\hline Body mass index & & & $26.7 \pm 2.6$ & & & $25.2 \pm 3.1$ & 0.095 \\
\hline Marital status & & & & & & & 0.089 \\
\hline Married & 22 & & & 23 & & & \\
\hline Single & 8 & & & 7 & & & \\
\hline Working condition & & & & & & & $<0.001$ \\
\hline Employed & 15 & & & 29 & & & \\
\hline Nonemployed & 15 & & & 1 & & & \\
\hline $\begin{array}{l}\text { Patient's global assessment } \\
\text { (0-100 mm VAS) }\end{array}$ & & & $5.0 \pm 2.2$ & & & $0.1 \pm 0.6$ & $<0.001$ \\
\hline Physician's global assessment & & & & & & & \\
\hline (0-100 mm VAS) & & & $4.4 \pm 2.1$ & & & $0.1 \pm 0.6$ & $<0.001$ \\
\hline Tender point count & & & $14.1 \pm 2.2$ & & & $0.2 \pm 0.9$ & $<0.001$ \\
\hline Fibromyalgia impact questionnaire & & & $46.5 \pm 0.9$ & & & $0.9 \pm 4.0$ & $<0.001$ \\
\hline Symptom severity scale & & & $8.6 \pm 1.8$ & & & $0.1 \pm 0.6$ & $<0.001$ \\
\hline Hamilton Anxiety Rating Scale & & & $11.7 \pm 8.9$ & & & $2.1 \pm 2.1$ & $<0.001$ \\
\hline Hamilton Depression Evaluation Scale & & & $8.4 \pm 3.8$ & & & $2.7 \pm 3.0$ & $<0.001$ \\
\hline Chronic widespread pain duration & & & $11.4 \pm 3.6$ & & & $0.3 \pm 1.0$ & $<0.001$ \\
\hline Frequency of anxiety & 21 & 70 & & 1 & 3.3 & & $<0.001$ \\
\hline Frequency of depression & 15 & 50 & & 1 & 3.3 & & $<0.001$ \\
\hline
\end{tabular}

Furthermore, no statistically significant differences in VA flow volume and velocity were noted between the two groups. However, a significant correlation between SS parameter and the $\mathrm{CBF}$ volume in FMS patients was determined.
Functional coupling between neural metabolism and $\mathrm{CBF}$ enables mapping of cerebral function according to hemodynamic response with neuroimaging methods, particularly functional magnetic resonance imaging. ${ }^{20} \mathrm{~A}$ number of

Table 2. Comparisons of radiological data parameters between fibromyalgia syndrom patients and healthy control groups

\begin{tabular}{|c|c|c|c|}
\hline & \multirow{2}{*}{$\frac{\text { Fibromyalgia syndrome patients }(\mathrm{n}=30)}{\text { Mean } \pm \mathrm{SD}}$} & \multirow{2}{*}{$\frac{\text { Healthy controls }(\mathrm{n}=30)}{\text { Mean } \pm \mathrm{SD}}$} & \multirow[b]{2}{*}{$p$} \\
\hline & & & \\
\hline Cerebral blood flow volume (mL/min) & $910.7 \pm 230.6$ & $889.3 \pm 182.4$ & 0.692 \\
\hline Right ICA volume (mL/min) & $364.0 \pm 116.4$ & $339.2 \pm 110.3$ & 0.400 \\
\hline Left ICA volume (mL/min) & $351.3 \pm 123.2$ & $354.0 \pm 106.6$ & 0.927 \\
\hline Right VA volume (mL/min) & $77.9 \pm 42.5$ & $86.6 \pm 47.1$ & 0.453 \\
\hline Left VA volume ( $\mathrm{mL} / \mathrm{min})$ & $117.5 \pm 54.2$ & $109.4 \pm 47.5$ & 0.543 \\
\hline Right ICA diameter $(\mathrm{mm})$ & $4.7 \pm 0.7$ & $4.6 \pm 0.7$ & 0.830 \\
\hline Left ICA diameter (mm) & $4.8 \pm 0.8$ & $4.6 \pm 0.7$ & 0.170 \\
\hline Right VA diameter (mm) & $3.2 \pm 0.6$ & $3.2 \pm 0.5$ & 0.774 \\
\hline Left VA diameter (mm) & $3.6 \pm 0.4$ & $3.5 \pm 0.5$ & 0.521 \\
\hline Right CCA mean peak systolic velocity $(\mathrm{cm} / \mathrm{sec})$ & $73.6 \pm 25.7$ & $77.1 \pm 17.5$ & 0.532 \\
\hline Left CCA mean peak systolic velocity $(\mathrm{cm} / \mathrm{sec})$ & $77.2 \pm 26.1$ & $81.7 \pm 19.7$ & 0.455 \\
\hline Right ICA mean peak systolic velocity $(\mathrm{cm} / \mathrm{sec})$ & $67.0 \pm 22.1$ & $68.3 \pm 19.8$ & 0.816 \\
\hline Left ICA mean peak systolic velocity $(\mathrm{cm} / \mathrm{sec})$ & $64.0 \pm 24.2$ & $72.5 \pm 22.8$ & 0.168 \\
\hline Right VA mean peak systolic velocity $(\mathrm{cm} / \mathrm{sec})$ & $31.2 \pm 9.9$ & $35.2 \pm 9.2$ & 0.104 \\
\hline Left VA mean peak systolic velocity $(\mathrm{cm} / \mathrm{sec})$ & $38.4 \pm 14.7$ & $36.5 \pm 9.5$ & 0.566 \\
\hline Right CCA end diastolic velocity $(\mathrm{cm} / \mathrm{sec})$ & $16.9 \pm 8.2$ & $16.7 \pm 5.4$ & 0.918 \\
\hline Left CCA end diastolic velocity $(\mathrm{cm} / \mathrm{sec})$ & $18.6 \pm 8.5$ & $17.3 \pm 6.9$ & 0.517 \\
\hline Right ICA end diastolik velocity $(\mathrm{cm} / \mathrm{sec})$ & $31.3 \pm 35.2$ & $21.4 \pm 8.2$ & 0.139 \\
\hline Left ICA end diastolic velocity $(\mathrm{cm} / \mathrm{sec})$ & $20.6 \pm 11.4$ & $22.0 \pm 7.2$ & 0.584 \\
\hline Right VA end diastolic velocity $(\mathrm{cm} / \mathrm{sec})$ & $8.3 \pm 3.6$ & $9.6 \pm 4.8$ & 0.268 \\
\hline Left VA end diastolic velocity $(\mathrm{cm} / \mathrm{sec})$ & $11.6 \pm 5.9$ & $9.6 \pm 3.3$ & 0.120 \\
\hline
\end{tabular}


functional magnetic resonance imaging studies have investigated the local distribution patterns of $\mathrm{CBF}$ in patients with FMS during somatosensory and pain processing. FMS patients, when compared to healthy equivalents, exhibit a lower threshold of pressure stimulation for blood flow response in a large part of the nociceptive matrix. ${ }^{21}$ Under nonpainful warmth stimulation, patients with FMS exhibit an enhanced blood flow response in the prefrontal and supplementary motor cortices, insula, and anterior cingulate. ${ }^{22}$ In patients with FMS, moderate pressure stimulation results in a blood flow increase in the sensorymotor cortex, insula, cingulate, and basal ganglia; in contrast, this response is almost completely restricted to the somatosensory cortex in healthy controls. ${ }^{23}$ Brain mapping studies have provided substantial insight into the neural basis of pain augmentation occurring in FMS. However, in addition to spatial distribution patterns, it may be worthwhile noting the dynamic dimension of the $\mathrm{CBF}$ response related to nociception. This is justified, in particular, by the well-documented time-dependence of interindividual differences in hemodynamic response. ${ }^{24-26}$

The close relationship between brain function, $\mathrm{CBF}$, and cerebral metabolism at rest has been the topic of interest for a number of researchers. ${ }^{3,27,28}$ In studies of both community and clinic groups, fibromyalgia has been strongly associated with anxiety and depressive symptoms, with approximately one-third of patients reporting major current problems with anxiety or depression. ${ }^{16,29,30} \mathrm{CBF}$ during acute anxiety is dependent on the interplay between central (arousal) and peripheral (sympathetic tone, carbon dioxide, etc.) factors. Extensive literature regarding the psychophysiology of anxiety indicates that wide variations exist between and within subjects. Indeed, it is likely that this will also prove to be true for cerebral circulation. Therefore, changes in $\mathrm{CBF}$ associated with anxiety are likely to depend on a number of these factors. ${ }^{31}$ To our knowledge, only one other study has evaluated CBF using transcranial color duplex Doppler ultrasonography in the literature. Duschek et al. ${ }^{32}$ reported that anterior cerebral artery blood flow increases in patients with FMS, when compared to controls. However, they did not evaluate the ICA or VA. It is well known that $\mathrm{CBF}$ and $\mathrm{CBF}$ velocity exhibit an increase during mental activity, and it is hypothesized that patients with FMS generally show increased brain activity; consequently, an increased blood supply and greater flow volume may be expected. ${ }^{33}$ On the one hand, Chen et al. ${ }^{27}$ reported that $\mathrm{CBF}$ decreases in patients with FMS. Variability of CBF in patients with FMS might be related to the variance in imaging methods and coexisting diseases. We observed in this study that the $\mathrm{CBF}$ volume and flow rates of FMS patients were not significantly higher than those of normal subjects, despite the fact that $50 \%$ of patients with fibromyalgia had depression and $70 \%$ had anxiety. These results may arise from a variety of secondary hemodynamic factors in relation to chronic anxiety and depression in fibromyalgia patients. Furthermore, it may be due to our small patient population. Therefore, further research is required including larger sample of fibromyalgia patients.

We evaluated the relationship between the magnitude of the hemodynamic response and the severity of clinical pain, depression and anxiety in patients with FMS. We observed a significant correlation between $\mathrm{CBF}$ volume and SS scale indicating disease severity in fibromyalgia patients. However, in this study, CBF volume was not correlated with emotional disturbance related with HAM-A and HAM-D, and functional disability related with FIQ. In addition to frequent depressive signs in panic disorder, preexisting somatic symptoms, including morning stiffness, depression, fatigue, insomnia, and reduced mental performance in FMS, may account for higher scores in FMS patients. However, the interplay between these symptoms and $\mathrm{CBF}$ volume remains unclear. $\mathrm{CBF}$ volume may be related to the temporal progression of FMS or to FMSmediated insufficiencies in the cardiovascular and pulmonary system, which may alter the heart rate and respiratory rate. Further research focused on clarifying these relationships is required.

Although many methods have been used to evaluate $\mathrm{CBF}$ in psychiatric patients, color duplex sonography is non-invasive, non-radioactive and easily applicable, making it an attractive option for quantifying $\mathrm{CBF}$ volume. However, a number of limitations exist in sonographic $\mathrm{CBF}$ volume measurements. Notably, the reliability of sonographic examination depends greatly on the skill and experience of the examiner. Furthermore, in patients with high carotid artery bifurcation, 
ICA plaques, stenosis, or an abnormal ICA morphology (kinking or tortuosity), sonographic CBF measurements cannot be performed. ${ }^{34}$

This study has some limitations. First, the number of participants in both groups was not determined by power analysis before the study. Second, an additional patient control group would be beneficial for the study. Finally, radiological measurements were made by only one researcher who was not blinded.

In conclusion, patients with FMS exhibited no increase in $\mathrm{CBF}$ volume, ICA flow, or VA flow, and $\mathrm{CBF}$ volume only correlated with $\mathrm{SS}$ scale among other clinical parameters reflecting disease severity. Color duplex Doppler ultrasonography is a non-invasive and easily applicable method, which assesses reliably the global $\mathrm{CBF}$ volume, and it should be considered as a primary methodology for further FMS studies. CBF volume may be associated with the clinical progression and features of FMS, and further research is required to examine these relationships.

\section{Declaration of conflicting interests}

The authors declared no conflicts of interest with respect to the authorship and/or publication of this article.

\section{Funding}

The authors received no financial support for the research and/or authorship of this article.

\section{REFERENCES}

1. Staud R. Heart rate variability as a biomarker of fibromyalgia syndrome. Fut Rheumatol 2008;3:475-83.

2. Arnold LM, Crofford LJ, Martin SA, Young JP, Sharma $U$. The effect of anxiety and depression on improvements in pain in a randomized, controlled trial of pregabalin for treatment of fibromyalgia. Pain Med 2007;8:633-8.

3. Raichle ME, Grubb RL Jr, Gado MH, Eichling JO, TerPogossian MM. Correlation between regional cerebral blood flow and oxidative metabolism. In vivo studies in man. Arch Neurol 1976;33:523-6.

4. Mathew RJ, Wilson WH. Anxiety and cerebral blood flow. Am J Psychiatry 1990;147:838-49.

5. Faravelli C, Marinoni M, Spiti R, Ginanneschi A, Serena A, Fabbri $C$, et al. Abnormal brain hemodynamic responses during passive orthostatic challenge in panic disorder. Am J Psychiatry 1997;154:378-83.
6. Reiman EM, Raichle ME, Robins E, Butler FK, Herscovitch P, Fox P, et al. The application of positron emission tomography to the study of panic disorder. Am J Psychiatry 1986;143:469-77.

7. De Cristofaro MT, Sessarego A, Pupi A, Biondi F, Faravelli C. Brain perfusion abnormalities in drugnaive, lactate-sensitive panic patients: a SPECT study. Biol Psychiatry 1993;33:505-12.

8. Doepp F, Schreiber SJ, Brunecker P, Valdueza JM. Ultrasonographic assessment of global cerebral blood volume in healthy adults. J Cereb Blood Flow Metab 2003;23:972-7.

9. Mazziotta JC. PET scanning: principles applications. Discussions Neurosci 1985;11:21-3.

10. Mathew RJ, Wilson WH. Cerebral blood flow changes induced by $\mathrm{CO} 2$ in anxiety. Psychiatry Res 1988;23:285-94.

11. Edvinsson L. Sympathetic control of cerebral circulation. Trends Neurosci 1982;5:425-29.

12. Gecici O, Acar M, Haktanir A, Emul M, Demirel $\mathrm{R}$, Yücel A, et al. Evaluation of cerebral blood flow volume using color duplex sonography in patients with untreated panic disorder. Psychiatry Clin Neurosci 2005;59:676-82.

13. Guedj E, Taieb D, Cammilleri S, Lussato D, de Laforte $C$, Niboyet $\mathrm{J}$, et al. $99 \mathrm{mTc}-\mathrm{ECD}$ brain perfusion SPECT in hyperalgesic fibromyalgia. Eur J Nucl Med Mol Imaging 2007;34:130-4.

14. Guedj E, Cammilleri S, Niboyet J, Dupont P, Vidal E, Dropinski JP, et al. Clinical correlate of brain SPECT perfusion abnormalities in fibromyalgia. $\mathrm{J}$ Nucl Med 2008;49:1798-803.

15. Guedj E, Cammilleri S, Niboyet J, Mundler O. Clinical image: Brain perfusion single-photonemission computed tomography findings in a patient with an asymmetric fibromyalgia syndrome. Arthritis Rheum 2009;60:298.

16. Wolfe F, Smythe HA, Yunus MB, Bennett RM, Bombardier C, Goldenberg DL, et al. The American College of Rheumatology 1990 Criteria for the Classification of Fibromyalgia. Report of the Multicenter Criteria Committee. Arthritis Rheum 1990;33:160-72.

17. Wolfe F, Clauw DJ, Fitzcharles MA, Goldenberg DL, Katz RS, Mease P, et al. The American College of Rheumatology preliminary diagnostic criteria for fibromyalgia and measurement of symptom severity. Arthritis Care Res (Hoboken) 2010;62:600-10.

18. Hamilton M. The assessment of anxiety states by rating. Br J Med Psychol 1959;32:50-5.

19. Hamilton M. A rating scale for depression. J Neurol Neurosurg Psychiatry 1960;23:56-62.

20. Logothetis NK, Pauls J, Augath M, Trinath T, Oeltermann A. Neurophysiological investigation of the basis of the fMRI signal. Nature 2001;412:150-7.

21. Gracely RH, Petzke F, Wolf JM, Clauw DJ. Functional magnetic resonance imaging evidence of augmented pain processing in fibromyalgia. Arthritis Rheum 
2002:46:1333-43.

22. Cook DB, Lange G, Ciccone DS, Liu WC, Steffener $\mathrm{J}$, Natelson $\mathrm{BH}$. Functional imaging of pain in patients with primary fibromyalgia. J Rheumatol 2004;31:364-78.

23. Pujol J, López-Solà M, Ortiz H, Vilanova JC, Harrison $\mathrm{BJ}$, Yücel M, et al. Mapping brain response to pain in fibromyalgia patients using temporal analysis of FMRI. PLoS One 2009;4:5224.

24. Sheth SA, Nemoto M, Guiou MW, Walker MA, Toga AW. Spatiotemporal evolution of functional hemodynamic changes and their relationship to neuronal activity. J Cereb Blood Flow Metab 2005;25:830-41.

25. Schuepbach D, Boeker H, Duschek S, Hell D. Rapid cerebral hemodynamic modulation during mental planning and movement execution: evidence of time-locked relationship with complex behavior. Clin Neurophysiol 2007;118:2254-62.

26. Duschek S, Schuepbach D, Schandry R. Timelocked association between rapid cerebral blood flow modulation and attentional performance. Clin Neurophysiol 2008;119:1292-9.

27. Chen JJ, Wang JY, Chang YM, Su SY, Chang CT, Sun SS, et al. Regional cerebral blood flow between primary and concomitant fibromyalgia patients: a possible way to differentiate concomitant fibromyalgia from the primary disease. Scand $J$ Rheumatol
2007;36:226-32.

28. Foerster BR, Petrou M, Harris RE, Barker PB, Hoeffner EG, Clauw DJ, et al. Cerebral blood flow alterations in pain-processing regions of patients with fibromyalgia using perfusion MR imaging. AJNR Am J Neuroradiol 2011;32:1873-8.

29. Wolfe F, Ross K, Anderson J, Russell IJ, Hebert L. The prevalence and characteristics of fibromyalgia in the general population. Arthritis Rheum 1995;38:19-28.

30. Reiman EM, Raichle ME, Robins E, Mintun MA, Fusselman MJ, Fox PT, et al. Neuroanatomical correlates of a lactate-induced anxiety attack. Arch Gen Psychiatry 1989;46:493-500.

31. Mathew RJ, Wilson WH. Anxiety and cerebral blood flow. Am J Psychiatry 1990;147:838-49.

32. Duschek S, Mannhart T, Winkelmann A, Merzoug K, Werner NS, Schuepbach D, et al. Cerebral blood flow dynamics during pain processing in patients with fibromyalgia syndrome. Psychosom Med 2012;74:802-9.

33. Guedj E. Neuroimaging findings in fibromyalgia: what clinical impact? Joint Bone Spine 2009;76:224-6.

34. Acar M, Degirmenci B, Yucel A, Albayrak R, Haktanir A. An evaluation of internal carotid artery and cerebral blood flow volume using color duplex sonography in patients with vertebral artery hypoplasia. Eur J Radiol 2005;53:450-3. 